\title{
Análisis de la Educación Intercultural en Grupos de Estudiantes de la Universidad Francisco de Paula Santander, en Colombia, que Fueron Víctimas del Conflicto Armado
}

\author{
Gerson Rueda, Luisa S. Paz y William R. Avendaño* \\ Facultad de Ciencias Empresariales, Universidad Francisco de Paula Santander, Cúcuta, Colombia. \\ (e-mail: gersonruedavera@ufps.edu.co; luisastellapm@ufps.edu.co; williamavendano@ufps.edu.co)
}

${ }^{*}$ Autor a quien debe ser dirigida la correspondencia

Recibido Ago. 28, 2018; Aceptado Oct. 29, 2018; Versión final Dic. 20, 2018, Publicado Ago. 2019

\section{Resumen}

Se analiza la forma en que la educación intercultural es asumida por docentes universitarios de la universidad Francisco de Paula Santander de Colombia adscritos a una facultad de ciencias empresariales, frente a una población estudiantil afectada por el conflicto armado. Se consideraron cuatro variables: conocimiento sobre educación intercultural, políticas institucionales, aspectos de consideración de los docentes en términos de multiculturalidad y prácticas de educación intercultural. Se aplicó una encuesta a un grupo de 105 docentes y 420 estudiantes apoyada en el paradigma empírico-analítico, el enfoque cuantitativo y un diseño no experimental/descriptivo. Los resultados muestran que los docentes no tienen un conocimiento claro en torno a la educación intercultural ni de las particularidades socio-culturales de los estudiantes. Se concluye que lo ideal sería reunir esfuerzos para reconocer todas las características de la población culturalmente diversa y reconocer las adecuaciones en materia de políticas, currículo y pedagogía para hacer coherente las acciones dentro del aula, de acuerdo a las necesidades de los estudiantes.

\section{Analysis of Intercultural Education of a group of Students at the Francisco de Paula Santander University, in Colombia, that were Victims of the Armed Conflict}

\begin{abstract}
The way in which intercultural education is assumed by university professors from the university Francisco de Paula Santander in Colombia belonging to the faculty of business who deal with a group of the student directly affected by the Colombian armed conflict. Four variables were considered: knowledge about intercultural education, institutional policies, aspects of consideration of teachers in terms of multiculturalism and intercultural education practices. A survey was applied to a group of 105 teachers and 420 students supported by the empirical-analytical paradigm, the quantitative approach and a non-experimental / descriptive design. The results show that teachers do not have a clear knowledge about intercultural education or the socio-cultural particularities of the student. It is concluded that an ideal situation would be to gather efforts to recognize all the characteristics of the culturally diverse population and recognize the adjustments in policy, curriculum and pedagogy to make coherent actions within the classroom, according to the needs of the students.
\end{abstract}




\section{INTRODUCCIÓN}

Para la sociedad colombiana, el conflicto armado no internacional ha implicado una verdadera tragedia extendida por más de 50 años, y por tanto, no resulta extraño que este conflicto armado interno sea uno de los principales temas dentro de la literatura académica y científica. Múltiples áreas del conocimiento han destinado esfuerzos para el análisis de los diversos componentes, elementos, factores o realidades asociadas con este fenómeno, destacándose la psicología, el trabajo social, la política, la economía, el derecho, la historia, y las ciencias sociales en general (Bergquist et al., 2001; Franco et al., 2006; González y Molinares, 2010; González, 2010). Estas investigaciones muestran diversas perspectivas y visiones sobre el conflicto armado en Colombia, algunas más sombrías y oscuras, otras más optimistas y esperanzadoras, pero todas con indicadores, cifras e información que facilita la tarea de identificar las posibilidades y los obstáculos para el Estado colombiano ante escenarios futuros de postconflicto.

El Grupo de Memoria Histórica en Colombia (2013) ha logrado sistematizar un cúmulo importante de información relacionada con el conflicto armado interno. Su labor ha permitido caracterizar las dinámicas del conflicto armado en el país, los actores involucrados, las tácticas y formas de enfrentamiento, el número aproximado de víctimas, las posibilidades de cambio y transformación, entre otras. Además, hasta el año 2012 se había presentado en Colombia 27.023 secuestros, 150.000 asesinatos, 1.344 individuos afectados por acciones bélicas, 1.566 personas afectadas por atentados terroristas, 11.751 personas víctimas de 1.982 casos de masacres, 25.007 eventos de desaparición forzada, 1.754 casos de violencia sexual. Estos datos corresponden a períodos que van desde la década de 1970 hasta el año 2012. Además, Colombia es uno de los países con mayor número de personas en condición de desplazamiento junto con Siria (7'947.655), Irak (4'026.863), República Democrática del Congo (3'616.882) y Sudán (2’498.776) (ACNUR, 2015).

Sin embargo, no se tiene plena certeza ni claridad sobre la cantidad de personas víctimas de este flagelo. De acuerdo con la Unidad para la Atención y Reparación Integral de Víctimas entre los años 1997 y 2011 se produjo en Colombia el desplazamiento de 3'943.509 individuos que agrupaban 923.218 familias (Moscoso y Burneo, 2014), pero conforme con la información de la Consultoría para los Derechos Humanos y el Desplazamiento (CODHES, 2011), desde el año 1985 hasta el 2011, el número de víctimas por desplazamiento forzado ha sido cercana a 5.3 millones. El Alto Comisionado de las Naciones Unidas para los Refugiados (ACNUR, 2015), describe que en ese año se había contabilizado 6'044.552 de personas en situación de desplazamiento, ubicando a Colombia como el segundo país con mayor número de refugiados a nivel mundial. En todo caso, las personas en situación de desplazamiento hacen frente a todo un conjunto de problemas que surgen con ocasión del destierro tanto de orden material y económico (la falta de acceso de recursos, limitaciones para encontrar empleo, condiciones precarias de vivienda, entre otras), como de naturaleza interna y personal al desarrollar una angustia emocional que les impide un pleno desarrollo cognitivo y social (Peltonen y Punamäki, 2010).

Como se observa, hay un avance significativo en cuanto a cifras y efectos del conflicto, pero la literatura no resulta tan amplia o diversa en cuanto al complejo binomio educación-conflicto armado/violencia. En otras palabras, no se han destinado esfuerzos significativos para que desde la cientificidad de la educación se construya una visión que permita la comprensión e interpretación del conflicto y la violencia en Colombia, y su incidencia en los factores asociados con la enseñanza y el aprendizaje, y las posibilidades de respuesta desde la escuela y la universidad. Y si bien es cierto, la universidad debe guardar una cierta neutralidad, la misma configura un espacio dinámico donde se construyen, socializan, exponen y transforman ideas, y en ese orden, "ser neutral no significa aislarse de los problemas, sino ganar distancia intelectual para pensar las mejores soluciones" (Lamus, 1999). En consecuencia, la universidad no puede ser ajena al conflicto armado interno y sus efectos, y por el contrario, debe adoptar una posición que le permita de manera objetiva identificar su papel dentro de la sociedad de manera activa y positiva (Pachón, 2018).

Uno de los fenómenos que la universidad como institución socio-política debe abordar desde su quehacer (docencia, investigación y extensión a la comunidad) es la integración de sus estudiantes, reconociendo que se trata de una comunidad caracterizada por la pluralidad, las diferencias y la multiculturalidad. Y sí se atiende a que muchos de los estudiantes son víctimas del conflicto armado, afectados por el desplazamiento forzado u otras formas de victimización, no hay duda de que la universidad debe considerar estos elementos al momento de diseñar y resignificar su direccionamiento estratégico, políticas, estrategias y acciones. Estas deben promover la inclusión y la adaptación de los estudiantes con realidades y subjetividades diversas, pues en su mayoría, estos provienen de espacios rurales, territorios con particularidades específicas (Beltrán, et al., 2015).

La universidad se enfrenta a la necesidad de materializar la inclusión como mecanismo de lucha contra la exclusión social, la mejora de la participación de los sujetos y la sensibilización en torno a la importancia del 
reconocimiento de los otros. Una esfera de acción relevante para el logro de estos objetivos es la docencia, espacio en el que se pueden introducir estrategias de inclusión partiendo de la diversidad cultural inherente a los estudiantes y a sus necesidades sociales. Desde esta perspectiva de inclusión, la cultura se interpreta como un factor esencial de comprensión. La inmersión en nuevos espacios urbanos de las personas en situación de desplazamiento trae consigo un encuentro de formas culturales e historias de vida, es decir, acumulados de fragmentos de origen diferente que no implica una transformación de la cultura abajo-arriba como lo describen Naranjo y Hurtado (2002). Estos autores conciben la cultura como un conjunto de elementos que no se encuentran estructurados bajo un sistema, y en esa medida, la introducción de un nuevo elemento no implica la modificación de los otros ya presentes. Esto sucede en el desarrollo de situaciones muy diversas como los viajes, los encuentros, las migraciones forzadas o los desastres, y ante estos escenarios, hay historias de vida que se trasladan y movilizan produciendo continuidades y discontinuidades, además de esfuerzos por conservar la identidad cultural y potenciar la participación en sociedad. Ante esto, se debe hacer énfasis "en el individuo, en la familia o el grupo local y en sus esfuerzos de transformación", pues se presentan acciones y estrategias de resistencia cultural que implican conflictos, negociaciones, cambios, invenciones y reconstrucciones.

La educación intercultural es una posible respuesta a estas dinámicas y diversidades culturales, además del reto de inclusión. La educación intercultural, también bajo las denominaciones educación multicultural, transcultural, global y/o de desarrollo (Dervin, 2015), en un primer momento hará referencia al bilingüismo y a la formación de grupos étnicos, pero su alcance se extenderá para la comprensión e interpretación de otras realidades y situaciones, como la de grupos humanos con diversidad de origen, marcados por el desplazamiento forzado o creencias religiosas distintas (López, 1997; Osuna, 2012; Jackson, 2014). Por ello no resulta extraño que Ferrao (2010) mencione que la educación intercultural en América Latina haya comprendido en las últimas décadas todo un proceso caracterizado por la complejidad, pluralidad y originalidad, llevando la educación o formación indígena a un ámbito de discusión que relaciona diversos proyectos de Estado y de la sociedad.

La educación intercultural es una práctica educativa con la que se quiere responder a la diversidad de grupos culturales para una mejor convivencia e inclusión participativa. Este enfoque forma parte de un interés internacional por tener en cuenta diferentes expresiones culturales en los procesos educativos (Osuna, 2012); sin embargo, no hay que confundirse: no todas las prácticas educativas enfocadas a la pluralidad o la aceptación de la diferencia, son interculturales. Aguado (1991) sostiene que la educación intercultural es un enfoque de tipo educativo que se sustenta en la diversidad cultural y promueve su respeto y valoración, y en esa medida, este enfoque resulta inclusivo al considerar a todos los miembros de una sociedad: "propone un modelo de intervención, formal e informal, holístico, integrado, configurador de todas las dimensiones del proceso educativo en orden a lograr la igualdad de oportunidades/resultados, la superación del racismo en sus diversas manifestaciones, la comunicación y competencia interculturales".

La literatura sobre el objeto de análisis demuestra que la educación intercultural resulta un tema de debate y necesaria reflexión (Osuna, 2012; Hajisoteriou, 2012; Tarozzi, 2014; Dervin, 2015). Entre otras cosas, los estudios disponibles demuestran que este enfoque ha sido implementado para dar respuesta a grupos sociales diversos, considerando criterios como el origen étnico y el bilingüismo, entre otros. Asimismo, algunas de estas investigaciones describen que aún faltan estudios empíricos en cuanto a métodos, estrategias y acciones a implementar, y coinciden en la necesidad de formar docentes con capacidades necesarias para concretar dentro del aula el llamado enfoque de la educación intercultural. Este último punto resulta crucial: la forma como los docentes abordan la educación intercultural depende de factores como la preparación intercultural, el conocimiento sobre el enfoque, el reconocimiento de la diversidad que hay dentro de los sujetos a formar, la organización curricular, y las políticas institucionales, entre otros factores.

Asimismo, los antecedentes en la materia muestran que el enfoque de la educación intercultural ha llevado al desarrollo del concepto competencia intercultural en los docentes, aunque se critica su empleo por tener origen dentro del discurso neoliberal y limitar la naturaleza de la educación intercultural. En consecuencia, Tarozzi (2014) y Sleeter (2014) coinciden en afirmar que el enfoque de la educación intercultural debe estar ligado con procesos emancipatorios que brinden a los sujetos posibilidades de empoderamiento para una mejor "participación pública constructiva como ciudadanos en una sociedad diversa que está luchando con las cuestiones de equidad". En otras palabras, la educación intercultural puede ayudar en la construcción de proyectos políticos de justicia social con gran alcance a públicos diversos y que beneficia a los mismos en términos de equidad y respeto de los derechos humanos, brindando un discurso opuesto al de la educación neoliberal y un referente de interpretación alternativa de la ciudadanía.

El encuentro cultural se manifiesta en diferentes momentos y situaciones: el contacto con el barrio o la zona de ubicación, el diálogo con autoridades y personas de la zona a la que llegan, las escuelas, las 
universidades, las empresas o los lugares de trabajo, entre otros. Allí se presenta una resistencia cultural como ya se ha señalado y ello exige un enfoque hacia el sujeto o individuo que facilite su adaptación sin que ello implique una pérdida de las formas culturales, y, por el contrario, se propicie un enriquecimiento personal con apertura a la participación y contribución social. Una interpretación adecuada de las culturas diversas que convergen en un espacio como la universidad puede servir de instrumento potencial para los procesos de enseñanza y aprendizaje (Cornejo, 2008). En este marco, la educación cumple un papel esencial y significativo, pues sirve de dispositivo en la reducción del impacto que tiene el conflicto armado y el desplazamiento sobre los sujetos, además de abrir nuevas posibilidades y propiciar transformaciones desde la pedagogía de la esperanza (Poveda, 2011).

Ante estas realidades, la educación intercultural constituye una posibilidad y alternativa, en especial, si se considera la escuela y la universidad como espacios de reproducción y transformación cultural, escenarios de socialización permanente en donde confluyen formas culturales y resistencias (Herrera, et al., 2016). La educación intercultural no se trata de un concepto nuevo, por el contrario, encuentra su origen en la postguerra cuando se empieza a excluir los nacionalismos los cuales dejaban a un lado las culturas locales para dar paso a la atención de minorías y grupos excluidos sobre todo desde la década de 1960 (Aguado, 1991). Un antecedente importante en materia de educación intercultural y que puede estar relacionado con la investigación adelantada, es la recomendación $\mathrm{R}(84) 18$ presentada por el Comité de Ministros de Educación de Europa en 1984 a fin de considerar la dimensión intercultural y capacitar profesores hacia la comprensión internacional en contextos de inmigración (Pérez, 2006; Faas, Hajisoteriou y Angelides, 2014).

Para generar un ambiente educativo dentro de la universidad, se requiere explorar las capacidades y el reconocimiento que tienen los docentes en torno al enfoque de la educación intercultural y los estudiantes que presentan condiciones culturales diversas. Pero a su vez, se hace perentorio indagar por el conocimiento de los mismos estudiantes en torno a la multiculturalidad que les rodea y de la que también hacen parte. Educar interculturalmente o formar desde el enfoque de la educación intercultural es "desarrollar la construcción de una realidad común de convivencia, donde nadie se sienta en posesión de la verdad, en depositario o receptáculo único y universal de la verdad" (Sáenz, 2006). En otras palabras, la educación intercultural tiende a la inclusión de todos, a la eliminación de las formas de poder basadas en la diferencia cultural y en la creación de ambientes de convivencia con igualdad de condiciones y oportunidades para todos. Un elemento clave en la educación intercultural es el reconocimiento del otro y la cultura que enmarca su identidad y su particular comprensión del mundo. El objetivo central de la educación intercultural es "ayudar a los ciudadanos a construir su propia identidad y a apreciar la de los otros" y ello implica reconocer e interpretar sus valores, tradiciones, historias y experiencias "haciendo frente a los inevitables conflictos, enfrentamientos y tensiones" (Aguado, 1991).

\section{METODOLOGÍA}

La investigación se encuentra enmarcada en el paradigma empírico-analítico y el enfoque cuantitativo apoyado en un análisis de frecuencia. La muestra estuvo conformada por dos grupos de participantes a quienes se les aplicó un mismo instrumento: docentes y estudiantes de tres departamentos adscritos a la Facultad de Ciencias Empresariales de la Universidad Francisco de Paula Santander, Cúcuta, Colombia. En total, la población de docentes fue 144 divididos así: 33 del Departamento de Estudios Internacionales y de Frontera, 69 del Departamento de Ciencias Administrativas y 42 del Departamento de Ciencias Contables y Financiera. Para calcular la muestra de docentes se aplicó la siguiente fórmula con base en el método estadístico aleatorio simple:

$$
n=\frac{Z c^{2} \times P \times Q \times}{E^{2} \times(N-1)+Z c^{2} \times P \times Q}
$$

Donde:

$\begin{array}{lll}\mathrm{Zc}= & 95 \% \text { 0 1.96 } & \text { Nivel de certeza, bajo la curva normal } \\ \mathrm{P}= & 0,5 & \text { Probabilidad de éxito } \\ \mathrm{Q}= & 0,5 & \text { Probabilidad de fracaso } \\ \mathrm{E}= & 5 \%-0,05 & \text { Nivel de error } \\ \mathrm{N}=\quad 144 & \text { Población }\end{array}$

Al aplicar la fórmula, la muestra se cuantifica en 105 docentes, los cuales se distribuyeron así: 24 del Departamento de Estudios Internacionales y de Frontera (23\%), 50 del Departamento de Ciencias Administrativas (48\%) y 31 del Departamento de Ciencias Contables y Financiera (29\%). Para ejercer un punto de control se tuvieron en cuenta a los estudiantes de cada uno de los programas de la siguiente manera: 96 (23\%) estudiantes del Departamento de Estudios Internacionales y de Frontera, 202 (48\%) 
estudiantes del Departamento de Ciencias Administrativas y 122 (29\%) estudiantes del Departamento de Ciencias Contables y Financiera, para un total de 420 estudiantes.

Se utilizó una única encuesta con escalas Likert y de percepción tanto para la muestra de docentes como de estudiantes y estuvo estructurada por doce preguntas que abarcaban cuatro dimensiones: conocimiento sobre educación intercultural, políticas institucionales de inclusión de personas culturalmente diferentes, aspectos que consideran los docentes en términos de multiculturalidad de los estudiantes y prácticas de educación intercultural de los docentes. No hubo intervención del investigador en la aplicación del instrumento y se usó el correo electrónico como medio para su entrega y recibimiento. El procesamiento de los datos se hizo a través de una serie de etapas de recuento, clasificación y ordenamiento en tablas y esquematizados en gráficos de manera conjunta (resultados docentes y resultados estudiantes), seguido de un análisis, teniendo como base el referente teórico para la construcción de conocimiento (Méndez, 2001). Para efecto de esta investigación se utilizaron tablas, para el proceso de vaciado de datos se utilizó el programa Excel y la parte estadística se hizo con el software SPSS 2.1. La validez de la encuesta, la cual es entendida como el "grado en que un instrumento realmente mide la variable que pretende medir" (Hernández et al., 2010), se hizo a través de juicio de expertos quienes exploraron el contenido de esta. Los expertos fueron escogidos considerando estos criterios: contar con doctorado en el campo de la educación o ciencias afines, tener publicaciones científicas y evidenciar prestigio en su carrera. En cuanto a la confiabilidad del instrumento, fue determinada mediante el coeficiente de Alfa de Cronbach.

\section{RESULTADOS}

El reconocimiento de los docentes respecto de su entorno y las formas culturales que allí convergen resulta un aspecto fundamental, es decir, la educación intercultural solo tendrá cabida en la medida que se identifiquen manifestaciones culturales diversas que requieren de una acción pedagógica alternativa como lo es la educación intercultural. A su vez, la única forma de dar paso a la educación intercultural es conociendo sus fundamentos, estrategias y acciones. La Tabla 1 muestra los resultados del instrumento aplicado por cada una de las preguntas formuladas. Como se describió en la metodología, cada pregunta hace parte de una o varias dimensiones o variables de análisis: conocimiento sobre educación intercultural (aspecto valorado 1), políticas institucionales de inclusión de personas culturalmente diferentes (aspecto valorado 2, 8), aspectos que consideran los docentes en términos de multiculturalidad de los estudiantes (aspecto valorado $3,4,7,10$ ) y prácticas de educación intercultural de los docentes (aspecto valorado 3, 5, 6, 9, 11, 12).

Tabla 1: Resultados del instrumento aplicado

\begin{tabular}{|c|c|c|c|c|c|}
\hline \multirow{3}{*}{ Aspecto valorado } & \multirow{3}{*}{ Indicador } & \multicolumn{4}{|c|}{ Frecuencia por grupo } \\
\hline & & \multicolumn{2}{|c|}{ Estudiantes } & \multicolumn{2}{|c|}{ Docentes } \\
\hline & & $N^{\circ}$ & $\%$ & $N^{\circ}$ & $\%$ \\
\hline \multirow{5}{*}{$\begin{array}{l}\text { 1. Conocimiento respecto de la educación } \\
\text { intercultural de estudiantes y docentes }\end{array}$} & Muy bajo & 54 & 12,9 & 5 & 4,8 \\
\hline & Bajo & 130 & 31,0 & 20 & 19,0 \\
\hline & Ni bajo ni alto & 156 & 37,1 & 50 & 47,6 \\
\hline & Alto & 68 & 16,2 & 30 & 28,6 \\
\hline & Muy alto & 12 & 2,9 & 0 & 0,0 \\
\hline \multirow{5}{*}{$\begin{array}{l}\text { 2. Conocimiento de las políticas educativas } \\
\text { institucionales y su eficacia en la promoción de } \\
\text { igualdad de condiciones en el proceso educativo }\end{array}$} & Desconozco totalmente & 44 & 10,5 & 5 & 4,8 \\
\hline & Desconozco & 182 & 43,3 & 30 & 28,6 \\
\hline & Ni desconozco ni conozco & 84 & 20,0 & 25 & 23,8 \\
\hline & Conozco & 94 & 22,4 & 45 & 42,9 \\
\hline & Conozco totalmente & 16 & 3,8 & 0 & 0 \\
\hline \multirow{5}{*}{$\begin{array}{l}\text { 3. Frecuencia con que los docentes tienen en } \\
\text { cuenta la ubicación de residencia u origen de los } \\
\text { estudiantes para los trabajos extracurriculares } \\
\text { asignados }\end{array}$} & Nunca & 176 & 41,9 & 15 & 14,3 \\
\hline & Casi nunca & 104 & 24,8 & 30 & 28,6 \\
\hline & Algunas veces & 108 & 25,7 & 35 & 33,3 \\
\hline & Casi siempre & 24 & 5,7 & 20 & 19,0 \\
\hline & Siempre & 8 & 1,9 & 5 & 4,8 \\
\hline \multirow{5}{*}{$\begin{array}{l}\text { 4. Conocimiento de los docentes de las } \\
\text { condiciones socioeconómicas y culturales de los } \\
\text { estudiantes que integran la asignatura/materia } \\
\text { que orientan }\end{array}$} & Muy bajo & 152 & 36,2 & 0 & 0 \\
\hline & Bajo & 144 & 34,3 & 20 & 19,0 \\
\hline & Ni bajo ni alto & 72 & 17,1 & 50 & 47,7 \\
\hline & Alto & 40 & 9,5 & 35 & 33,3 \\
\hline & Muy alto & 12 & 2,9 & 0 & 0 \\
\hline \multirow{5}{*}{$\begin{array}{l}\text { 5. Perspectiva del aula como espacio de diálogo } \\
\text { sin restricciones por condición socioeconómica } \\
\text { o cultural del estudiante }\end{array}$} & Totalmente en desacuerdo & 52 & 12,4 & 0 & 0 \\
\hline & En desacuerdo & 62 & 14,8 & 5 & 4,8 \\
\hline & Ni en desacuerdo ni de acuerdo & 92 & 21,9 & 15 & 14,3 \\
\hline & De acuerdo & 140 & 33,3 & 45 & 42,9 \\
\hline & Totalmente de acuerdo & 74 & 17,6 & 40 & 38,1 \\
\hline
\end{tabular}


Tabla 1 (continuación)

\begin{tabular}{|c|c|c|c|c|c|}
\hline \multirow{3}{*}{ Aspecto valorado } & \multirow{3}{*}{ Indicador } & \multicolumn{4}{|c|}{ Frecuencia por grupo } \\
\hline & & \multicolumn{2}{|c|}{ Estudiantes } & \multicolumn{2}{|c|}{ Docentes } \\
\hline & & $N^{\circ}$ & $\%$ & $N^{\circ}$ & $\%$ \\
\hline \multirow{5}{*}{$\begin{array}{l}\text { 6. Frecuencia con que los docentes desarrollan } \\
\text { actividades extracurriculares (congresos, } \\
\text { seminarios, foros, integraciones y salidas) } \\
\text { teniendo en cuenta las condiciones } \\
\text { socioeconómicas y culturales de los estudiantes }\end{array}$} & Nunca & 72 & 17,1 & 10 & 9,5 \\
\hline & Casi nunca & 146 & 34,8 & 30 & 28,6 \\
\hline & Algunas veces & 162 & 38,6 & 40 & 38,1 \\
\hline & Casi siempre & 30 & 7,1 & 15 & 14,3 \\
\hline & Siempre & 10 & 2,4 & 10 & 9,5 \\
\hline \multirow{5}{*}{$\begin{array}{l}\text { 7. Conocimiento de los docentes en torno a la } \\
\text { diversidad de condiciones sociales de los } \\
\text { estudiantes }\end{array}$} & Muy Bajo & 56 & 13,3 & 10 & 9,5 \\
\hline & Bajo & 166 & 39,5 & 20 & 19,0 \\
\hline & Ni bajo Ni alto & 116 & 27,6 & 30 & 28,6 \\
\hline & Alto & 72 & 17,1 & 45 & 42,9 \\
\hline & Muy alto & 10 & 2,4 & 0 & 0 \\
\hline \multirow{5}{*}{$\begin{array}{l}\text { 8. Perspectiva sobre las políticas educativas } \\
\text { institucionales y la incorporación de la } \\
\text { diversidad cultural de los estudiantes }\end{array}$} & Totalmente en desacuerdo & 62 & 14,7 & 0 & 0 \\
\hline & En desacuerdo & 114 & 27,1 & 45 & 42,9 \\
\hline & $\mathrm{Ni}$ en desacuerdo ni de acuerdo & 186 & 44,3 & 45 & 42,9 \\
\hline & De acuerdo & 46 & 11,0 & 10 & 9,5 \\
\hline & Totalmente de acuerdo & 12 & 2,9 & 5 & 4,7 \\
\hline \multirow{5}{*}{$\begin{array}{l}\text { 9. Frecuencia con que los docentes tienen en } \\
\text { cuenta las condiciones sociales de los } \\
\text { estudiantes en el desarrollo que actividades }\end{array}$} & Nunca & 62 & 14.8 & 10 & 9.5 \\
\hline & Casi nunca & 134 & 31.9 & 10 & 9.5 \\
\hline & Algunas veces & 172 & 40.9 & 35 & 33.3 \\
\hline & Casi siempre & 40 & 9.5 & 30 & 28.6 \\
\hline & Siempre & 12 & 2.9 & 20 & 19.1 \\
\hline \multirow{5}{*}{$\begin{array}{l}\text { 10. Conocimiento que tienen los docentes de las } \\
\text { formas culturales de los estudiantes a su cargo }\end{array}$} & Muy bajo & 64 & 15.2 & 15 & 14.3 \\
\hline & Bajo & 170 & 40.5 & 20 & 19.0 \\
\hline & Ni bajo ni alto & 122 & 29.0 & 35 & 33.3 \\
\hline & Alto & 52 & 12.4 & 30 & 28.6 \\
\hline & Muy alto & 12 & 2.9 & 5 & 4.8 \\
\hline \multirow{5}{*}{$\begin{array}{l}\text { 11. Frecuencia en que los docentes permiten el } \\
\text { reconocimiento y discusión de las culturas y } \\
\text { particularidades socioculturales que se hacen } \\
\text { presenten a través de los estudiantes }\end{array}$} & Nunca & 70 & 16.6 & 9 & 8.5 \\
\hline & Casi nunca & 145 & 34,5 & 12 & 11.4 \\
\hline & Algunas veces & 168 & 40 & 43 & 40.9 \\
\hline & Casi siempre & 17 & 4.0 & 22 & 20.9 \\
\hline & Siempre & 10 & 2.3 & 19 & 18.1 \\
\hline \multirow{5}{*}{$\begin{array}{l}\text { 12. Nivel de sentimiento de los estudiantes de } \\
\text { contextos socioculturales diferentes, sobre que } \\
\text { tanto se sienten incluidos en el aula }\end{array}$} & Muy Bajo & 83 & 19.7 & 10 & 9.5 \\
\hline & Bajo & 91 & 21.6 & 11 & 10.4 \\
\hline & $\mathrm{Ni}$ bajo $\mathrm{Ni}$ alto & 190 & 45.2 & 33 & 31.4 \\
\hline & Alto & 22 & 5.2 & 38 & 36.1 \\
\hline & Muy alto & 24 & 5.7 & 13 & 12.3 \\
\hline
\end{tabular}

Los resultados consignados en la Tabla 1 permiten inferir respecto de la variable o dimensión conocimiento sobre educación intercultural que tanto estudiantes como docentes no tienen claridad en cuanto al enfoque de la educación intercultural y el concepto de interculturalidad. La distribución de participantes se encuentra agrupada para ambos grupos en las opciones muy bajo, bajo y ni alto ni bajo. Respecto de la variable o dimensión políticas institucionales de inclusión de personas culturalmente diferentes, los resultados indican que a nivel institucional no hay conocimiento sobre políticas que tengan por objeto la igualdad de condiciones en el proceso educativo. Docentes, y sobre todo estudiantes, señalan no tener conocimiento sobre las políticas educativas institucionales de este tipo (aspecto valorado 2). Dentro del aspecto valorado 8 se muestran los resultados sobre la percepción de los participantes en torno a las políticas educativas institucionales para la atención de la diversidad cultural en la universidad. Esta información complementa los hallazgos del aspecto valorado 2. En la opinión de los informantes no hay políticas institucionales claras que tengan como objeto la atención a la diversidad cultural dentro de la universidad.

En cuanto a la dimensión o variable aspectos que consideran los docentes en términos de multiculturalidad de los estudiantes se logra inferir varios aspectos. Primero, los docentes no consideran el lugar de origen y ubicación de los estudiantes en la planificación y desarrollo de actividades y trabajos extracurriculares, es decir, estos aspectos no sirven de criterio en la dirección del proceso de enseñanza (aspecto valorado 3 ). Segundo, los docentes en su mayoría no tienen conocimiento sobre las condiciones socioeconómicas y culturales de los estudiantes, lo cual se ve reflejado en el direccionamiento de la formación y perceptible a los estudiantes pues se puede verificar posiciones similares para ambos grupos (aspecto valorado 4). Esto se refleja en el aspecto valorado 7 donde se muestra de igual modo el conocimiento de los docentes en cuanto a la diversidad de condiciones sociales de los estudiantes: los mayores porcentajes se ubican en los criterios bajo/ ni bajo ni altos en relación con los estudiantes (cerca del $70 \%$ ), aunque este porcentaje se acerca al $50 \%$ en los docentes. Ambos datos permiten concluir que no hay un conocimiento de la diversidad 
de condiciones sociales de los estudiantes. Tercero, el aspecto valorado 10 indica que los docentes desde la opinión de los estudiantes tienen poca claridad sobre las formas culturales que enmarcan a los sujetos de formación de manera similar que las condiciones sociales y socioeconómicas. Lo mismo se indica en los resultados de docentes donde más del $65 \%$ indica escaso o nulo conocimiento de las formas culturales de los estudiantes.

Respecto de la variable o dimensión prácticas de educación intercultural de los docentes, los resultados evidencian varios aspectos a resaltar. Primero, resultados del aspecto valorado 3 ya analizado con anterioridad, indica que dentro de las prácticas docentes no se tiene en cuenta la ubicación de residencia 0 el lugar de origen de los estudiantes. Segundo, hay una división entre los informantes en cuanto a su perspectiva del aula como espacio de diálogo sin restricción por condición socioeconómica y cultural del estudiante. Cerca del $50 \%$ de los estudiantes consideran negativa esta afirmación, mientras que los demás perciben que el aula sí es un espacio de diálogo en el que la condición socioeconómica y/o cultural no es un factor de exclusión (aspecto valorado 5). Esta última idea también tiene mayor recepción dentro del grupo de docentes (80\%). Tercero, conforme a resultados del aspecto valorado 6, tanto estudiantes como docentes convergen en su posición: no se tiene en cuenta dentro del aula las condiciones socioeconómicas y culturales al momento de la planificación y desarrollo de actividades extracurriculares como congresos, seminarios, foros, integraciones y salidas. Cuarto, el aspecto valorado 9 complementa los hallazgos del aspecto 7. La opinión de los participantes se divide en esta pregunta sobre las condiciones sociales de los estudiantes como elemento que guía el desarrollo de las actividades. Para cerca del $45 \%$ de estudiantes nunca o casi nunca se tiene en cuenta las condiciones sociales y para $40 \%$ sólo algunas veces; por el contrario, $48 \%$ de los docentes considera que siempre o casi siempre se tienen en cuenta las condiciones sociales. Quinto, el aspecto valorado 11 refleja la frecuencia en que las particularidades de la diversidad cultural de los estudiantes es objeto de discusión y reflexión dentro del aula por disposición y mediación del docente. Los hallazgos son congruentes con los demás obtenidos: más del $40 \%$ de estudiantes consideran que nunca o casi nunca se posibilita este encuentro de reflexión intercultural y el $40 \%$ señala que sólo algunas veces. Por su parte, cerca del $40 \%$ de docentes señala que casi siempre lo siente. Concuerdan los resultados de un $40 \%$ de docentes con la opinión de los estudiantes en el indicador algunas veces. Y sexto, el aspecto valorado 12 puede interpretarse como un resumen de todos los hallazgos: $40 \%$ de los estudiantes no se sienten incluidos y $45.2 \%$ no refleja un sentimiento de inclusión satisfactorio. Estos resultados son inversos desde la perspectiva de los docentes: cerca del $48 \%$ de los docentes consideran que los estudiantes sienten que son incluidos.

\section{DISCUSIÓN}

La educación intercultural sólo puede tener cabida en la universidad sí se reconoce la misma como un espacio de socialización donde convergen manifestaciones culturales diversas, es decir, cada individuo aporta a la identidad global de la comunidad, y en esa medida, la educación intercultural como enfoque brinda un marco de comprensión y acción a los docentes. Sin embargo, para el caso de estudio, aunque hay un reconocimiento de la diversidad cultural en los programas adscritos a la Facultad de Ciencias Empresariales de la Universidad Francisco de Paula Santander, no hay un conocimiento preciso ni específico sobre la educación intercultural como enfoque ni de las políticas públicas que tienen por objeto el desarrollo de este para una educación igualitaria e inclusiva (variables: conocimiento sobre educación intercultural y políticas institucionales de inclusión de personas culturalmente diferentes). Esta situación incide de manera directa en el escaso reconocimiento de aspectos multiculturales y el desarrollo de estrategias por parte de los docentes considerando estos aspectos de multiculturalidad de los estudiantes.

Por lo anterior, resulta oportuno que la Universidad establezca una política perfectamente definida hacia este objetivo que sirva de base en las prácticas docentes, y eso incluye estrategias de capacitación y formación como bien lo expresan Fermoso (1992) en cuanto a competencias y habilidades para la educación intercultural: 1) potenciar habilidades comunicativas, tanto verbales como no verbales; 2) desarrollar la capacidad de entender la propia cultura desde la teoría y la práctica; 3) aceptación de la posibilidad de diferentes perspectivas culturales, 4) preparación para superar los prejuicios racistas y xenófobos; y 5) conocimiento de los fenómenos migratorios, los contextos culturales y las diversas formas de expresar los mismos sentimientos en cada cultura.Lo anterior permitirá que se mejoren los resultados relacionados con la dimensión o variable aspectos que consideran los docentes en términos de multiculturalidad de los estudiantes. Debido a que no se conocen los lugares de origen o de residencia, los aspectos sociales o económicos, o los aspectos culturales de los estudiantes, las competencias a desarrollar deben dirigirse a estrategias a través de las cuales se pueden aprehender a estas particularidades. Estos son algunos de los elementos que se deberían conocer de manera general en términos de multiculturalidad, aunque a partir de ello pueden surgir nuevas esferas de interés. En relación a los estudiantes víctimas del conflicto armado, resulta necesario identificar las particularidades culturales y los intereses que guían sus acciones en función de ampliar su participación, superar su exclusión social y económica, y visibilizar los aportes que pueden ofrecer a la construcción de espacios pacíficos y de ayuda. 
Ahora bien, respecto a la variable o dimensión prácticas de educación intercultural de los docentes, la ausencia de acciones y estrategias en materia de educación intercultural al interior de la Facultad obedece a varios factores: 1) La ausencia de políticas educativas institucionales que brinden un espacio de reflexión y acción al tema. 2) El poco conocimiento de los docentes sobre aquellas formas culturales que se hacen manifiestas dentro de los programas donde dirigen su asignatura. 3) La primacía de los planes de estudio y contenidos programáticos sobre la realidad de los estudiantes (contexto, vida, experiencias). 4) El poco conocimiento de las condiciones socioeconómicas de los estudiantes. Se deberá considerar en el desarrollo de las estrategias un conjunto de metas como lo menciona Galino y Escribano (1990) respecto de la educación intercultural: 1) promover la idea de la diversidad cultural y étnica; 2) familiarizar a cada grupo o individuo con las características de los otros; 3) proporcionar aspectos culturales diferenciales a los alumnos; 4) ayudarles a interesarse por dimensiones pertenecientes a otras culturas: música, literatura, estilo de vida; 5) iniciar en actitudes y destrezas intelectuales, sociales y emocionales que permitan situarse adecuadamente en una sociedad integrada; y 6) cambio no sólo ideológico sino en las relaciones políticas, económicas e internacionales que afectarían a todo el sistema educativo.

La universidad objeto de análisis no asume un papel protagónico con respecto a la población culturalmente diversa que asiste a las aulas. En cuanto a la población en condición de desplazamiento, se limita a garantizar su acceso y permanencia, pero no pone su situación en el ámbito de la reflexión pedagógica y educativa, el estigma que los ha marcado ni las particularidades culturales que los caracteriza por su origen. De allí que la cuestión socioeconómica sea un aspecto fundamental, porque esta población no cuenta con medios o recursos suficientes, y configura un criterio al momento de la planificación y el desarrollo de la enseñanza. Sin embargo, los resultados muestran desde la perspectiva de los estudiantes que no se tienen en cuenta las capacidades socioeconómicas de estos al momento de diseñar las actividades formativas tanto curriculares como extracurriculares. La poca claridad de los docentes en cuanto a la situación socioeconómica de los estudiantes y a la cultura que los caracteriza, hace que los procesos formativos se realicen de manera plana e inflexible. $Y$ en ese sentido, los docentes omiten de su reflexión pedagógica aquellas características de los universitarios y que son base esencial en los procesos de enseñanza. Por ello se afirma que prime la formalidad sobre la realidad, los objetivos de las asignaturas sobre las necesidades reales de los estudiantes. Sin duda alguna, se debe adelantar dentro de la Facultad y la universidad en general, una discusión sobre estas realidades como la de los estudiantes que han sido víctimas de desplazamiento forzado para que toda su experiencia se convierta en dispositivo de cambio y en problema que guíe su futura acción profesional.

\section{CONCLUSIONES}

Conforme a los resultados del estudio y su respectivo análisis y discusión, se obtienen las siguientes conclusiones: 1) los docentes y estudiantes de la institución objeto de estudio no tienen claridad sobre interculturalidad y la educación intercultural, 2) la institución analizada no cuenta con políticas de inclusión de personas culturalmente diferentes, 3) no se tiene precisión sobre los aspectos relacionados con la multiculturalidad como origen, residencia, características sociales o económicas, o particularidades culturales, y 4) hay un escaso desarrollo de estrategias o acciones por parte de los docentes en función de lograr una mayor inclusión partiendo de las diferencias socio-culturales de los estudiantes.

Respecto de lo anterior, se sugiere considerar la educación intercultural como estrategia de inclusión, en especial, para la población víctima del conflicto armado. En efecto, este enfoque ha tenido un auge en las últimas décadas y ha servido como enfoque para dar respuesta a necesidades o problemas específicos de población culturalmente diversa. Su aplicación al contexto de la población en condición de desplazamiento representa una potencialidad. Se recomienda aunar esfuerzos para reconocer todas las características de la población culturalmente diversa y reconocer las fallas a nivel de políticas, currículo, planes de estudio, modelos y métodos educativos y pedagógicos implementados para hacer coherente las acciones dentro del aula con los requerimientos de la población atendida.

\section{REFERENCIAS}

Aguado, M. T., La educación intercultural: concepto, paradigmas, realizaciones. en Lecturas de Pedagogía Diferencial, 1aㅡ. Ed., 89-104, Dykinson, Madrid, España (1991)

Alto Comisionado de las Naciones Unidas para los Refugiados (ACNUR), Mundo en guerra. Tendencias Globales 2014. Desplazamiento forzado en 2014, 1를., 2-5, ACNUR, Ginebra, Suiza (2015)

Beltrán, Y., Y. Martínez y A. Vargas, El sistema educativo colombiano en el camino hacia la inclusión. Avances y retos, doi: 10.5294/edu.2015.18.1.4, Educ. Educ., 18(1), 62-75 (2015)

Bergquist, C., R. Peñaranda y G. Sánchez, Violence in Colombia, 1990-2000: waging war and negotiating peace, $1^{a}$ Ed., 39-52, Rowman \& Littlefield Publishers, Wilmington, USA (2001) 
Consultoría para los Derechos Humanos y el Desplazamiento (CODHES), De la seguridad a la prosperidad democrática en medio del conflicto, 1르. Ed., 18-27, CODHES, Bogotá, Colombia (2011)

Cornejo, J., La historia, la cultura y el arte en la formación universitaria integral del docente de ciencias exactas y naturales, doi: 10.4067/S0718-50062008000100006, Form. Univ., 1(1), 37-46 (2008)

Dervin, F., Towards post-intercultural teacher education: analysing 'extreme' intercultural dialogue to reconstruct interculturality, doi: 10.1080/02619768.2014.902441, Euro J. Teach Edu., 38(1), 71-86 (2015)

Faas, D., C. Hajisoteriou y A. Panayiotis, Intercultural education in Europe: policies, practices and trends, doi: 10.1002/berj.3080, Br. Edu. Res. J., 40(2), 300-318 (2014)

Fermoso, P., Educación intercultural: la Europa sin fronteras, 1ª Ed., 129-156, Narcea, Madrid, España (1992)

Ferrao, V. M., Educación intercultural en América Latina: distintas concepciones y tensiones actuales, doi: 10.4067/S0718-07052010000200019, Est. Pedag., 36(2), 333-342 (2010)

Franco, S., C.M. Suarez y otros tres autores, The effects of the armed conflict on the life and health in Colombia, doi: 10.1590/S1413-81232006000200013, Ciênc Saúde Coletiva, 11(2), 349-361 (2006)

Galino, Á. y A. Escribano, La educación intercultural en el enfoque y desarrollo del curriculum, 1a Ed., 7-20, Narcea, Madrid, España (1990)

González, R. e I. Molinares, La violencia en Colombia. Una mirada particular para su comprensión. De cómo percibimos la violencia social a gran escala y hacemos invisible la violencia no mediática, Investig. Desarro., ISSN: 01213261,18(2), 346-369 (2010)

González, R., Conflicto y postconflicto colombiano en el ámbito internacional. En González, R., y A. C. Mason, Colombia y el hemisferio ante el nuevo orden global, $1^{\text {a }}$ Ed., 33-45, UniNorte, Barranquilla, Colombia (2010)

Grupo de Memoria Histórica, ¡Basta ya! Colombia: Memorias de guerra y dignidad, 1aㅡ Ed., 31-101, Centro Nacional de Memoria Histórica, Bogotá, Colombia (2013)

Hajisoteriou, C., Intercultural education set forward: operational strategies and procedures in Cypriot classrooms, doi: 10.1080/14675986.2012.686022, Intercultural Edu., 23(2), 133-146 (2012)

Hernández, R., C. Fernández y M. Baptista, Metodología de la Investigación, 5ª Ed., 52-65, Mcgraw-Hill, México D.F. (2010)

Herrera, C., C. Pérez y G. Echeita, Teorías implícitas y prácticas de enseñanza que promueven la inclusión educativa en la universidad. Instrumentos y antecedentes para la reflexión y discusión, doi: 10.4067/S0718-50062016000500006, Form. Univ., 9(5), 49-64 (2016)

Jackson, R., Signposts-Policy and practice for teaching about religions and non-religious world views in intercultural education, $1^{\text {a }}$ Ed., 13-26, Council of Europe, Estrasburgo, Francia (2014)

Lamus, D., La universidad y el conflicto armado, Reflexión Política, ISSN: 0124-0781, 1(2), 1-3 (1999)

López, L. E., La diversidad étnica, cultural y lingüística latinoamericana y los recursos humanos que la educación requiere, Rev. Iberoam. Edu., 13, 7-98 (1997)

Méndez, C. E., Metodología: Diseño y desarrollo del proceso de investigación con énfasis en ciencias empresariales, $4^{a}$ Ed., Limusa, Bogotá, Colombia (2006)

Moscoso, R. y N. Burneo, Más allá de las fronteras: la población colombiana en su proceso de integración urbana en la ciudad de Quito, 1a. Ed., 6-7, ACNUR, Quito, Ecuador (2014)

Naranjo, G. y D. Hurtado, El derecho a la ciudad: migrantes y desplazados en las ciudades colombianas, De Paso. Rostros y Caminos, (1), 4-15 (2002)

Osuna, C., En torno a la educación intercultural. Una revisión crítica, doi: 10-4438/1988-592X-RE-2012-358-182, Rev. Edu., 358, 38-58 (2012)

Pachón, W., Inclusión social de actores del conflicto armado colombiano: retos para la educación superior, doi: 10.12804/revistas.urosario.edu.co/desafios/a.4917, Desafíos, 30(1), 279-308 (2018)

Peltonen, K., y R. L. Punamäki, Preventive interventions among children exposed to trauma of armed conflict: a literature review, doi: 10.1002/ab.20334, Aggress Behav, 36(2), 95-116 (2010)

Pérez, J., Educación Intercultural: de las necesidades formativas del maestro a las respuestas institucionales para la atención a la diversidad, Tesis Doctoral, Servicio de Publicaciones de la Universidad de Almería, España (2006)

Poveda, A.C., Economic development, inequality and poverty: An analysis of urban violence in Colombia, doi: 10.1080/13600818.2011.620085, Oxford Development Studies, 39(4), 453-468 (2011)

Sáenz, R., La educación intercultural, Rev. Edu., 339, 859-881 (2006)

Sleeter, C.E., Multiculturalism and education for citizenship in a context of neoliberalism, doi: 10.1080/14675986.2014.886357, Intercultural Edu., 25(2), 85-94 (2014)

Tarozzi, M., Building an "intercultural ethos" in teacher education, doi: 10.1080/14675986.2014.888804, Intercultural Edu., 25(2), 128-142 (2014) 
